\title{
STANDING WAVE SOLUTIONS OF SCHRÖDINGER SYSTEMS WITH DISCONTINUOUS NONLINEARITY IN ANISOTROPIC MEDIA
}

TEODORA-LILIANA DINU

Received 5 July 2005; Revised 14 April 2006; Accepted 5 July 2006

We establish the existence of an entire solution for a class of stationary Schrödinger systems with subcritical discontinuous nonlinearities and lower bounded potentials that blow up at infinity. The proof is based on the critical point theory in the sense of Clarke and we apply Chang's version of the mountain pass lemma for locally Lipschitz functionals. Our result generalizes in a nonsmooth framework a result of Rabinowitz (1992) related to entire solutions of the Schrödinger equation.

Copyright (c) 2006 Hindawi Publishing Corporation. All rights reserved.

\section{Introduction and the main result}

The Schrödinger equation plays the role of Newton's laws and conservation of energy in classical mechanics, that is, it predicts the future behavior of a dynamic system. The linear form of Schrödinger's equation is

$$
\Delta \psi+\frac{8 \pi^{2} m}{\hbar^{2}}(E(x)-V(x)) \psi=0
$$

where $\psi$ is the Schrödinger wave function, $m$ is the mass, $\hbar$ denotes Planck's constant, $E$ is the energy, and $V$ stands for the potential energy. The structure of the nonlinear Schrödinger equation is much more complicated. This equation describes various phenomena arising in self-channelling of a high-power ultra-short laser in matter, in the theory of Heisenberg ferromagnets and magnons, in dissipative quantum mechanics, in condensed matter theory, in plasma physics (e.g., the Kurihara superfluid film equation). We refer to $[7,14]$ for a modern overview, including applications.

Consider the model problem

$$
i \hbar \psi_{t}=-\frac{\hbar^{2}}{2 m} \Delta \psi+V(x) \psi-\gamma|\psi|^{p-1} \psi \quad \text { in } \mathbb{R}^{N}(N \geq 2),
$$

Hindawi Publishing Corporation International Journal of Mathematics and Mathematical Sciences Volume 2006, Article ID 73619, Pages 1-13

DOI 10.1155/IJMMS/2006/73619 
where $p<2 N /(N-2)$ if $N \geq 3$ and $p<+\infty$ if $N=2$. In the study of this equation, Oh [12] supposed that the potential $V$ is bounded and possesses a nondegenerate critical point at $x=0$. More precisely, it is assumed that $V$ belongs to the class $\left(V_{a}\right)$ (for some $a$ ) introduced by Kato in [10]. Taking $\gamma>0$ and $\hbar>0$ sufficiently small and using a LyapunovSchmidt-type reduction, Oh [12] proved the existence of a standing wave solution of problem (1.2), that is, a solution of the form

$$
\psi(x, t)=e^{-i E t / \hbar} u(x) .
$$

Note that substituting the ansatz (1.3) into (1.2) leads to

$$
-\frac{\hbar^{2}}{2} \Delta u+(V(x)-E) u=|u|^{p-1} u
$$

The change of variable $y=\hbar^{-1} x$ (and replacing $y$ by $x$ ) yields

$$
-\Delta u+2\left(V_{\hbar}(x)-E\right) u=|u|^{p-1} u \quad \text { in } \mathbb{R}^{N},
$$

where $V_{\hbar}(x)=V(\hbar x)$.

In a celebrated paper, Rabinowitz [13] continued the study of standing wave solutions of nonlinear Schrödinger equations. After making a standing wave ansatz, Rabinowitz reduces the problem to that of studying the semilinear elliptic equation

$$
-\Delta u+b(x) u=f(x, u) \quad \text { in } \mathbb{R}^{N},
$$

under suitable conditions on $b$ and assuming that $f$ is smooth, superlinear, and subcritical.

Inspired by Rabinowitz' paper, we consider the following class of coupled elliptic systems in $\mathbb{R}^{N}(N \geq 3)$ :

$$
\begin{array}{ll}
-\Delta u_{1}+a(x) u_{1}=f\left(x, u_{1}, u_{2}\right) & \text { in } \mathbb{R}^{N} \\
-\Delta u_{2}+b(x) u_{2}=g\left(x, u_{1}, u_{2}\right) & \text { in } \mathbb{R}^{N}
\end{array}
$$

We point out that coupled nonlinear Schrödinger systems describe some physical phenomena such as the propagation in birefringent optical fibers or Kerr-like photorefractive media in optics. Another motivation to the study of coupled Schrödinger systems arises from the Hartree-Fock theory for the double condensate, that is, a binary mixture of Bose-Einstein condensates in two different hyperfine states (cf. [5]). System (1.7) is also important for industrial applications in fiber communications systems [8] and all-optical switching devices [9].

Throughout this paper, we assume that $a, b \in L_{\mathrm{loc}}^{\infty}\left(\mathbb{R}^{N}\right)$ and there exist $\underline{a}, \underline{b}>0$ such

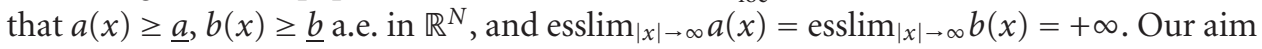
in this paper is to study the existence of solutions to the above problem in the case when $f, g$ are not continuous functions. Our goal is to show how variational methods can be used to find existence results for stationary nonsmooth Schrödinger systems. 
Throughout this paper, we assume that $f(x, \cdot, \cdot), g(x, \cdot, \cdot) \in L_{\mathrm{loc}}^{\infty}\left(\mathbb{R}^{2}\right)$. Denote

$$
\begin{aligned}
& \underline{f}\left(x, t_{1}, t_{2}\right)=\lim _{\delta \rightarrow 0} \operatorname{essinf}\left\{f\left(x, s_{1}, s_{2}\right) ;\left|t_{i}-s_{i}\right| \leq \delta ; i=1,2\right\}, \\
& \bar{f}\left(x, t_{1}, t_{2}\right)=\lim _{\delta \rightarrow 0} \operatorname{esssup}\left\{f\left(x, s_{1}, s_{2}\right) ;\left|t_{i}-s_{i}\right| \leq \delta ; i=1,2\right\}, \\
& \underline{g}\left(x, t_{1}, t_{2}\right)=\lim _{\delta \rightarrow 0} \operatorname{essinf}\left\{g\left(x, s_{1}, s_{2}\right) ;\left|t_{i}-s_{i}\right| \leq \delta ; i=1,2\right\}, \\
& \bar{g}\left(x, t_{1}, t_{2}\right)=\lim _{\delta \rightarrow 0} \operatorname{esssup}\left\{g\left(x, s_{1}, s_{2}\right) ;\left|t_{i}-s_{i}\right| \leq \delta ; i=1,2\right\} .
\end{aligned}
$$

Under these conditions, we reformulate problem (1.7) as follows:

$$
\begin{array}{ll}
-\Delta u_{1}+a(x) u_{1} \in\left[\underline{f}\left(x, u_{1}(x), u_{2}(x)\right), \bar{f}\left(x, u_{1}(x), u_{2}(x)\right)\right] & \text { a.e. } x \in \mathbb{R}^{N}, \\
-\Delta u_{2}+b(x) u_{2} \in\left[\underline{g}\left(x, u_{1}(x), u_{2}(x)\right), \bar{g}\left(x, u_{1}(x), u_{2}(x)\right)\right] & \text { a.e. } x \in \mathbb{R}^{N} .
\end{array}
$$

Let $H^{1}=H\left(\mathbb{R}^{N}, \mathbb{R}^{2}\right)$ denote the Sobolev space of all $U=\left(u_{1}, u_{2}\right) \in\left(L^{2}\left(\mathbb{R}^{N}\right)\right)^{2}$ with weak derivatives $\partial u_{1} / \partial x_{j}, \partial u_{2} / \partial x_{j}(j=1, \ldots, N)$ also in $L^{2}\left(\mathbb{R}^{N}\right)$, endowed with the usual norm

$$
\|U\|_{H_{1}}^{2}=\int_{\mathbb{R}^{N}}\left(|\nabla U|^{2}+|U|^{2}\right) d x=\int_{\mathbb{R}^{N}}\left(\left|\nabla u_{1}\right|^{2}+\left|\nabla u_{2}\right|^{2}+u_{1}^{2}+u_{2}^{2}\right) d x .
$$

Given the functions $a, b: \mathbb{R}^{N} \rightarrow \mathbb{R}$ as above, define the subspace

$$
E=\left\{U=\left(u_{1}, u_{2}\right) \in H^{1} ; \int_{\mathbb{R}^{N}}\left(\left|\nabla u_{1}\right|^{2}+\left|\nabla u_{2}\right|^{2}+a(x) u_{1}^{2}+b(x) u_{2}^{2}\right) d x<+\infty\right\} .
$$

Then the space $E$ endowed with the norm

$$
\|U\|_{E}^{2}=\int_{\mathbb{R}^{N}}\left(\left|\nabla u_{1}\right|^{2}+\left|\nabla u_{2}\right|^{2}+a(x) u_{1}^{2}+b(x) u_{2}^{2}\right) d x
$$

becomes a Hilbert space.

Since $a(x) \geq \underline{a}>0, b(x) \geq \underline{b}>0$, we have the continuous embeddings $H^{1} \hookrightarrow L^{q}\left(\mathbb{R}^{N}, \mathbb{R}^{2}\right)$ for all $2 \leq q \leq 2^{*}=2 N /(N-2)$.

We assume throughout the paper that $f, g: \mathbb{R}^{N} \times \mathbb{R}^{2} \rightarrow \mathbb{R}$ are nontrivial measurable functions satisfying the following hypotheses:

$$
\begin{array}{ll}
|f(x, t)| \leq C\left(|t|+|t|^{p}\right) & \text { for a.e. }(x, t) \in \mathbb{R}^{N} \times \mathbb{R}^{2}, \\
|g(x, t)| \leq C\left(|t|+|t|^{p}\right) & \text { for a.e. }(x, t) \in \mathbb{R}^{N} \times \mathbb{R}^{2},
\end{array}
$$

where $p<2 *$;

$$
\begin{aligned}
& \lim _{\delta \rightarrow 0} \operatorname{esssup}\left\{\frac{|f(x, t)|}{|t|} ;(x, t) \in \mathbb{R}^{N} \times(-\delta,+\delta)^{2}\right\}=0, \\
& \lim _{\delta \rightarrow 0} \operatorname{esssup}\left\{\frac{|g(x, t)|}{|t|} ;(x, t) \in \mathbb{R}^{N} \times(-\delta,+\delta)^{2}\right\}=0 ;
\end{aligned}
$$


4 Schrödinger systems with discontinuous nonlinearity

$f$ and $g$ are chosen so that the mapping $F: \mathbb{R}^{N} \times \mathbb{R}^{2} \rightarrow \mathbb{R}$ defined by $F\left(x, t_{1}, t_{2}\right):=\int_{0}^{t_{1}} f(x$, $\left.\tau, t_{2}\right) d \tau+\int_{0}^{t_{2}} g(x, 0, \tau) d \tau$ satisfies

$$
F\left(x, t_{1}, t_{2}\right)=\int_{0}^{t_{2}} g\left(x, t_{1}, \tau\right) d \tau+\int_{0}^{t_{1}} f(x, \tau, 0) d \tau, \quad F\left(x, t_{1}, t_{2}\right)=0 \quad \text { iff } t_{1}=t_{2}=0 ;
$$

there exists $\mu>2$ such that for any $x \in \mathbb{R}^{N}$,

$$
0 \leq \mu F\left(x, t_{1}, t_{2}\right) \leq \begin{cases}t_{1} \underline{f}\left(x, t_{1}, t_{2}\right)+t_{2} \underline{g}\left(x, t_{1}, t_{2}\right), & t_{1}, t_{2} \in[0,+\infty), \\ t_{1} \underline{f}\left(x, t_{1}, t_{2}\right)+t_{2} \bar{g}\left(x, t_{1}, t_{2}\right), & t_{1} \in[0,+\infty), t_{2} \in(-\infty, 0], \\ t_{1} \bar{f}\left(x, t_{1}, t_{2}\right)+t_{2} \bar{g}\left(x, t_{1}, t_{2}\right), & t_{1}, t_{2} \in(-\infty, 0], \\ t_{1} \bar{f}\left(x, t_{1}, t_{2}\right)+t_{2} \underline{g}\left(x, t_{1}, t_{2}\right), & t_{1} \in(-\infty, 0], t_{2} \in[0,+\infty) .\end{cases}
$$

Definition 1.1. A function $U=\left(u_{1}, u_{2}\right) \in E$ is called solution to the problem (1.9) if there exists a function $W=\left(w_{1}, w_{2}\right) \in L^{2}\left(\mathbb{R}^{N}, \mathbb{R}^{2}\right)$ such that

(i)

$$
\begin{array}{ll}
\underline{f}\left(x, u_{1}(x), u_{2}(x)\right) \leq w_{1}(x) \leq \bar{f}\left(x, u_{1}(x), u_{2}(x)\right) & \text { a.e. } x \text { in } \mathbb{R}^{N}, \\
\underline{g}\left(x, u_{1}(x), u_{2}(x)\right) \leq w_{2}(x) \leq \bar{g}\left(x, u_{1}(x), u_{2}(x)\right) & \text { a.e. } x \text { in } \mathbb{R}^{N}
\end{array}
$$

(ii)

$$
\begin{gathered}
\int_{\mathbb{R}^{N}}\left(\nabla u_{1} \nabla v_{1}+\nabla u_{2} \nabla v_{2}+a(x) u_{1} v_{1}+b(x) u_{2} v_{2}\right) d x \\
=\int_{\mathbb{R}^{N}}\left(w_{1} v_{1}+w_{2} v_{2}\right) d x, \quad \forall\left(v_{1}, v_{2}\right) \in E .
\end{gathered}
$$

Our main result is the following.

Theorem 1.2. Assume that conditions (1.13)-(1.16) are fulfilled. Then problem (1.9) has at least a nontrivial solution in $E$.

\section{Auxiliary results}

We first recall some basic notions from the Clarke gradient theory for locally Lipschitz functionals (see $[3,4]$ for more details). Let $E$ be a real Banach space and assume that $I: E \rightarrow \mathbb{R}$ is a locally Lipschitz functional. Then the Clarke generalized gradient is defined by

$$
\partial I(u)=\left\{\xi \in E^{*} ; I^{0}(u, v) \geq\langle\xi, v\rangle, \forall v \in E\right\},
$$

where $I^{0}(u, v)$ stands for the directional derivative of $I$ at $u$ in the direction $v$, that is,

$$
I^{0}(u, v)=\limsup _{\substack{w \rightarrow u \\ \lambda \succ 0}} \frac{I(w+\lambda v)-I(w)}{\lambda} .
$$


Let $\Omega$ be an arbitrary domain in $\mathbb{R}^{N}$. Set

$$
E_{\Omega}=\left\{U=\left(u_{1}, u_{2}\right) \in H^{1}\left(\Omega ; \mathbb{R}^{2}\right) ; \int_{\Omega}\left(\left|\nabla u_{1}\right|^{2}+\left|\nabla u_{2}\right|^{2}+a(x) u_{1}^{2}+b(x) u_{2}^{2}\right) d x<+\infty\right\},
$$

which is endowed with the norm

$$
\|U\|_{E_{\Omega}}^{2}=\int_{\Omega}\left(\left|\nabla u_{1}\right|^{2}+\left|\nabla u_{2}\right|^{2}+a(x) u_{1}^{2}+b(x) u_{2}^{2}\right) d x .
$$

Then $E_{\Omega}$ becomes a Hilbert space.

Lemma 2.1. The functional $\Psi_{\Omega}: E_{\Omega} \rightarrow \mathbb{R}, \Psi_{\Omega}(U)=\int_{\Omega} F(x, U) d x$ is locally Lipschitz on $E_{\Omega}$. Proof. We first observe that

$$
\begin{aligned}
F(x, U) & =F\left(x, u_{1}, u_{2}\right) \\
& =\int_{0}^{u_{1}} f\left(x, \tau, u_{2}\right) d \tau+\int_{0}^{u_{2}} g(x, 0, \tau) d \tau \\
& =\int_{0}^{u_{2}} g\left(x, u_{1}, \tau\right) d \tau+\int_{0}^{u_{1}} f(x, \tau, 0) d \tau
\end{aligned}
$$

is a Carathéodory functional which is locally Lipschitz with respect to the second variable. Indeed, by (1.13),

$$
\begin{aligned}
\left|F\left(x, t_{1}, t\right)-F\left(x, s_{1}, t\right)\right| & =\left|\int_{s_{1}}^{t_{1}} f(x, \tau, t) d \tau\right| \\
& \leq\left|\int_{s_{1}}^{t_{1}} C\left(|\tau, t|+|\tau, t|^{p}\right) d \tau\right| \\
& \leq k\left(t_{1}, s_{1}, t\right)\left|t_{1}-s_{1}\right| .
\end{aligned}
$$

Similarly,

$$
\left|F\left(x, t, t_{2}\right)-F\left(x, t, s_{2}\right)\right| \leq k\left(t_{2}, s_{2}, t\right)\left|t_{2}-s_{2}\right|
$$

Therefore,

$$
\begin{aligned}
\left|F\left(x, t_{1}, t_{2}\right)-F\left(x, s_{1}, s_{2}\right)\right| & \leq\left|F\left(x, t_{1}, t_{2}\right)-F\left(x, s_{1}, t_{2}\right)\right|+\left|F\left(x, t_{1}, s_{2}\right)-F\left(x, s_{1}, s_{2}\right)\right| \\
& \leq k(V)\left|\left(t_{2}, s_{2}\right)-\left(t_{1}, s_{1}\right)\right|,
\end{aligned}
$$

where $V$ is a neighborhood of $\left(t_{1}, t_{2}\right),\left(s_{1}, s_{2}\right)$.

Set

$$
\chi_{1}(x)=\max \left\{u_{1}(x), v_{1}(x)\right\}, \quad \chi_{2}(x)=\max \left\{u_{2}(x), v_{2}(x)\right\}, \quad \forall x \in \Omega
$$


6 Schrödinger systems with discontinuous nonlinearity

It is obvious that if $U=\left(u_{1}, u_{2}\right), V=\left(v_{1}, v_{2}\right)$ belong to $E_{\Omega}$, then $\left(\chi_{1}, \chi_{2}\right) \in E_{\Omega}$. So, by Hölder's inequality and the continuous embedding $E_{\Omega} \subset L^{p}\left(\Omega ; \mathbb{R}^{2}\right)$,

$$
\left|\Psi_{\Omega}(U)-\Psi_{\Omega}(V)\right| \leq C\left(\left\|\chi_{1}, \chi_{2}\right\|_{E_{\Omega}}\right)\|U-V\|_{E_{\Omega}}
$$

which concludes the proof.

The following result is a generalization of [11, Lemma 6].

Lemma 2.2. Let $\Omega$ be an arbitrary domain in $\mathbb{R}^{N}$ and let $f: \Omega \times \mathbb{R}^{2} \rightarrow \mathbb{R}$ be a Borel function such that $f(x, \cdot) \in L_{\text {loc }}^{\infty}\left(\mathbb{R}^{2}\right)$. Then $\underline{f}$ and $\bar{f}$ are Borel functions.

Proof. Since the requirement is local, we may suppose that $f$ is bounded by $M$ and it is nonnegative. Denote

$$
f_{m, n}\left(x, t_{1}, t_{2}\right)=\left(\int_{t_{1}-1 / n}^{t_{1}+1 / n} \int_{t_{2}-1 / n}^{t_{2}+1 / n}\left|f\left(x, s_{1}, s_{2}\right)\right|^{m} d s_{1} d s_{2}\right)^{1 / m} .
$$

Since $\bar{f}\left(x, t_{1}, t_{2}\right)=\lim _{\delta \rightarrow 0} \operatorname{esssup}\left\{f\left(x, s_{1}, s_{2}\right) ;\left|t_{i}-s_{i}\right| \leq \delta ; i=1,2\right\}$, we deduce that for every $\varepsilon>0$, there exists $n \in \mathbb{N}^{*}$ such that for $\left|t_{i}-s_{i}\right|<1 / n(i=1,2)$, we have $\mid \operatorname{esssup} f\left(x, s_{1}\right.$, $\left.s_{2}\right)-\bar{f}\left(x, t_{1}, t_{2}\right) \mid<\varepsilon$ or, equivalently,

$$
\bar{f}\left(x, t_{1}, t_{2}\right)-\varepsilon<\operatorname{esssup} f\left(x, s_{1}, s_{2}\right)<\bar{f}\left(x, t_{1}, t_{2}\right)+\varepsilon .
$$

By the second inequality in (2.12), we obtain

$$
f\left(x, s_{1}, s_{2}\right) \leq \bar{f}\left(x, t_{1}, t_{2}\right)+\varepsilon \quad \text { a.e. } x \in \Omega \text { for }\left|t_{i}-s_{i}\right|<\frac{1}{n} \quad(i=1,2)
$$

which yields

$$
f_{m, n}\left(x, t_{1}, t_{2}\right) \leq\left(\bar{f}\left(x, t_{1}, t_{2}\right)+\varepsilon\right)\left(\sqrt{\frac{4}{n^{2}}}\right)^{1 / m}
$$

Let

$$
A=\left\{\left(s_{1}, s_{2}\right) \in \mathbb{R}^{2} ;\left|t_{i}-s_{i}\right|<\frac{1}{n}(i=1,2) ; \bar{f}\left(x, t_{1}, t_{2}\right)-\varepsilon \leq f\left(x, s_{1}, s_{2}\right)\right\} .
$$

By the first inequality in (2.12) and the definition of the essential supremum, we obtain that $|A|>0$ and

$$
f_{m, n} \leq\left(\iint_{A}\left(f\left(x, s_{1}, s_{2}\right)\right)^{m} d s_{1} d s_{2}\right)^{1 / m} \geq\left(\bar{f}\left(x, s_{1}, s_{2}\right)-\varepsilon\right)|A|^{1 / m} .
$$

Since (2.14) and (2.16) imply that

$$
\bar{f}\left(x, t_{1}, t_{2}\right)=\lim _{n \rightarrow \infty} \lim _{m \rightarrow \infty} f_{m, n}\left(x, t_{1}, t_{2}\right),
$$


it suffices to prove that $f_{m, n}$ is Borel. Let

$$
\begin{gathered}
\mathcal{M}=\left\{f: \Omega \times \mathbb{R}^{2} \longrightarrow \mathbb{R} ;|f| \leq M \text { and } f \text { is a Borel function }\right\}, \\
\mathcal{N}=\left\{f \in \mathcal{M} ; f_{m, n} \text { is a Borel function }\right\} .
\end{gathered}
$$

$\mathcal{M}$ is the smallest set of functions having the following properties (cf. [1, page 178]):

(i) $\left\{f \in C\left(\Omega \times \mathbb{R}^{2} ; \mathbb{R}\right) ;|f| \leq M\right\} \subset M$;

(ii) $f^{(k)} \in \mathcal{M}$ and $f^{(k)} \stackrel{k}{\rightarrow} f$ imply that $f \in M$.

Since $\mathcal{N}$ contains obviously the continuous functions and (ii) is also true for $\mathcal{N}$, then by the Lebesgue dominated convergence theorem, we obtain that $\mathcal{M}=\mathcal{N}$. For $\underline{f}$, we note that $\underline{f}=-(-\bar{f})$ and the proof of Lemma 2.2 is complete.

Let us now assume that $\Omega \subset \mathbb{R}^{N}$ is a bounded domain. By the continuous embedding $L^{p+1}\left(\Omega ; \mathbb{R}^{2}\right) \hookrightarrow L^{2}\left(\Omega ; \mathbb{R}^{2}\right)$, we may define the locally Lipchitz functional $\Psi_{\Omega}: L^{p+1}\left(\Omega ; \mathbb{R}^{2}\right) \rightarrow$ $\mathbb{R}$ by $\Psi_{\Omega}(U)=\int_{\Omega} F(x, U) d x$.

LEMMA 2.3. Under the above assumptions and for any $U \in L^{p+1}\left(\Omega ; \mathbb{R}^{2}\right)$,

$$
\partial \Psi_{\Omega}(U)(x) \subset[\underline{f}(x, U(x)), \bar{f}(x, U(x))] \times[\underline{g}(x, U(x)), \bar{g}(x, U(x))] \text { a.e. } x \text { in } \Omega,
$$

in the sense that if $W=\left(w_{1}, w_{2}\right) \in \partial \Psi_{\Omega}(U) \subset L^{p+1}\left(\Omega ; \mathbb{R}^{2}\right)$, then

$$
\begin{array}{ll}
\underline{f}(x, U(x)) \leq w_{1}(x) \leq \bar{f}(x, U(x)) & \text { a.e. } x \text { in } \Omega, \\
\underline{g}(x, U(x)) \leq w_{2}(x) \leq \bar{g}(x, U(x)) & \text { a.e. } x \text { in } \Omega .
\end{array}
$$

Proof. By the definition of the Clarke gradient, we have

$$
\int_{\Omega}\left(w_{1} v_{1}+w_{2} v_{2}\right) d x \leq \Psi_{\Omega}^{0}(U, V) \quad \forall V=\left(v_{1}, v_{2}\right) \in L^{p+1}\left(\Omega ; \mathbb{R}^{2}\right) .
$$

Choose $V=(v, 0)$ such that $v \in L^{p+1}(\Omega), v \geq 0$ a.e. in $\Omega$. Thus, by Lemma 2.2,

$$
\begin{aligned}
\int_{\Omega} w_{1} v & \leq \limsup _{\substack{\left(h_{1}, h_{2}\right) \rightarrow U \\
\lambda \succ 0}} \frac{\int_{\Omega}\left(\int_{h_{1}(x)}^{h_{1}(x)+\lambda v(x)} f\left(x, \tau, h_{2}(x)\right) d \tau\right) d x}{\lambda} \\
& \leq \int_{\Omega}\left(\limsup _{\substack{\left(h_{1}, h_{2}\right) \rightarrow U \\
\lambda \succ 0}} \frac{1}{\lambda} \int_{h_{1}(x)}^{h_{1}(x)+\lambda v(x)} f\left(x, \tau, h_{2}(x)\right) d \tau\right) d x \\
& \leq \int_{\Omega} \bar{f}\left(x, u_{1}(x), u_{2}(x)\right) v(x) d x .
\end{aligned}
$$

Analogously, we obtain

$$
\int_{\Omega} \underline{f}\left(x, u_{1}(x), u_{2}(x)\right) v(x) d x \leq \int_{\Omega} w_{1} v d x \quad \forall v \geq 0 \text { in } \Omega .
$$


Arguing by contradiction, suppose that (2.20) is false. Then there exist $\varepsilon>0$, a set $A \subset \Omega$ with $|A|>0$, and $w_{1}$ as above such that

$$
w_{1}(x)>\bar{f}(x, U(x))+\varepsilon \quad \text { in } A .
$$

Taking $v=\mathbf{1}_{A}$ in (2.23), we obtain

$$
\int_{\Omega} w_{1} v d x=\int_{A} w_{1} d x \leq \int_{A} \bar{f}(x, U(x)) d x,
$$

which contradicts (2.25). Proceeding in the same way, we obtain the corresponding result for $g$ in (2.21).

By Lemma 2.3, Chang [2, Lemma 2.1], and the embedding $E_{\Omega} \hookrightarrow L^{p+1}\left(\Omega, \mathbb{R}^{2}\right)$, we obtain also that for $\Psi_{\Omega}: E_{\Omega} \rightarrow \mathbb{R}, \Psi_{\Omega}(U)=\int_{\Omega} F(x, U) d x$, we have

$$
\partial \Psi_{\Omega}(U)(x) \subset[\underline{f}(x, U(x)), \bar{f}(x, U(x))] \times[\underline{g}(x, U(x)), \bar{g}(x, U(x))] \quad \text { a.e. } x \in \Omega .
$$

Let $V \in E_{\Omega}$. Then $\tilde{V} \in E$, where $\tilde{V}: \mathbb{R}^{N} \rightarrow \mathbb{R}^{2}$ is defined by

$$
\tilde{V}= \begin{cases}V(x) & x \text { in } \Omega, \\ 0 & \text { otherwise. }\end{cases}
$$

For $W \in E^{*}$, we consider $W_{\Omega} \in E_{\Omega}^{*}$ such that $\left\langle W_{\Omega}, V\right\rangle=\langle W, \tilde{V}\rangle$ for all $V$ in $E_{\Omega}$. Set $\Psi: E \rightarrow \mathbb{R}, \Psi(U)=\int_{\mathbb{R}^{N}} F(x, U)$.

Lemma 2.4. Let $W \in \partial \Psi(U)$, where $U \in E$. Then $W_{\Omega} \in \partial \Psi_{\Omega}(U)$, in the sense that $W_{\Omega} \in$ $\partial \Psi_{\Omega}\left(\left.U\right|_{\Omega}\right)$.

Proof. By the definition of the Clarke gradient, we deduce that $\langle W, \tilde{V}\rangle \leq \Psi^{0}(U, \tilde{V})$ for all $V$ in $E_{\Omega}$,

$$
\begin{aligned}
\Psi^{0}(U, \tilde{V}) & =\limsup _{\substack{H \rightarrow U, H \in E \\
\lambda \rightarrow 0}} \frac{\Psi(H+\lambda \tilde{V})-\Psi(H)}{\lambda} \\
& =\limsup _{\substack{H \rightarrow U, H \in E \\
\lambda \rightarrow 0}} \frac{\int_{\mathbb{R}^{N}}(F(x, H+\lambda \tilde{V})-F(x, H)) d x}{\lambda} \\
& =\limsup _{\substack{H \rightarrow U, H \in E \\
\lambda \rightarrow 0}} \frac{\int_{\Omega}(F(x, H+\lambda \tilde{V})-F(x, H)) d x}{\lambda} \\
& =\limsup _{\substack{H \rightarrow U, H \in E_{\Omega} \\
\lambda \rightarrow 0}} \frac{\int_{\Omega}(F(x, H+\lambda \tilde{V})-F(x, H)) d x}{\lambda} \\
& =\Psi_{\Omega}^{0}(U, V) .
\end{aligned}
$$

Hence $\left\langle W_{\Omega}, V\right\rangle \leq \Psi_{\Omega}^{0}(U, V)$, which implies that $W_{\Omega} \in \partial \Psi_{\Omega}^{0}(U)$. 
By Lemmas 2.3 and 2.4, we obtain that for any $W \in \partial \Psi(U)$ (with $U \in E$ ), $W_{\Omega}$ satisfies (2.20) and (2.21). We also observe that for $\Omega_{1}, \Omega_{2} \subset \mathbb{R}^{N}$, we have $\left.W_{\Omega_{1}}\right|_{\Omega_{1} \cap \Omega_{2}}=$ $W_{\Omega_{2}} \mid \Omega_{1} \cap \Omega_{2}$.

Let $W_{0}: \mathbb{R}^{N} \rightarrow \mathbb{R}$, where $W_{0}(x)=W_{\Omega}(x)$ if $x \in \Omega$. Then $W_{0}$ is well defined and

$$
W_{0}(x) \in[\underline{f}(x, U(x)), \bar{f}(x, U(x))] \times[\underline{g}(x, U(x)), \bar{g}(x, U(x))] \quad \text { a.e. } x \in \mathbb{R}^{N},
$$

and for all $\varphi \in C_{c}^{\infty}\left(\mathbb{R}^{N}, \mathbb{R}^{2}\right),\langle W, \varphi\rangle=\int_{\mathbb{R}^{N}} W_{0} \varphi$. By density of $C_{c}^{\infty}\left(\mathbb{R}^{N}, \mathbb{R}^{2}\right)$ in $E$, we deduce that $\langle W, V\rangle=\int_{\mathbb{R}^{N}} W_{0} V d x$ for all $V$ in $E$. Hence

$$
W(x)=W_{0}(x) \in[\underline{f}(x, U(x)), \bar{f}(x, U(x))] \times[\underline{g}(x, U(x)), \bar{g}(x, U(x))] \quad \text { a.e. } x \in \mathbb{R}^{N} .
$$

\section{Proof of Theorem 1.2}

Define the energy functional $I: E \rightarrow \mathbb{R}$ by

$$
\begin{aligned}
I(U) & =\frac{1}{2} \int_{\mathbb{R}^{N}}\left(\left|\nabla u_{1}\right|^{2}+\left|\nabla u_{2}\right|^{2}+a(x) u_{1}^{2}+b(x) u_{2}^{2}\right) d x-\int_{\mathbb{R}^{N}} F(x, U) d x \\
& =\frac{1}{2}\|U\|_{E}^{2}-\Psi(U) .
\end{aligned}
$$

The existence of solutions to problem (1.9) will be justified by a nonsmooth variant of the mountain pass theorem (see [2]) applied to the functional $I$, even if the PS condition is not fulfilled. More precisely, we check the following geometric hypotheses:

$$
\begin{gathered}
I(0)=0, \quad \exists V \in E, \quad \text { such that } I(V) \leq 0 ; \\
\exists \beta, \rho>0 \quad \text { such that } I \geq \beta \text { on }\left\{U \in E ;\|U\|_{E}=\rho\right\} .
\end{gathered}
$$

Verification of (3.2). It is obvious that $I(0)=0$. For the second assertion, we need the following lemma.

Lemma 3.1. There exist two positive constants $C_{1}$ and $C_{2}$ such that

$$
f(x, s, 0) \geq C_{1} s^{\mu-1}-C_{2}, \quad \text { for a.e. } x \in \mathbb{R}^{N} ; s \in[0,+\infty) .
$$

Proof. We first observe that (1.16) implies that

$$
0 \leq \mu F(x, s, 0) \leq \begin{cases}s \underline{f}(x, s, 0), & s \in[0,+\infty), \\ s \bar{f}(x, s, 0), & s \in(-\infty, 0]\end{cases}
$$

which places us in the conditions of [11, Lemma 5]. 
Verification of (3.2) continued. Choose $v \in C_{c}^{\infty}\left(\mathbb{R}^{N}\right)-\{0\}$ so that $v \geq 0$ in $\mathbb{R}^{N}$. We have $\int_{\mathbb{R}^{N}}|\nabla v|^{2}+a(x) v^{2}<\infty$, hence $t(v, 0) \in E$ for all $t \in \mathbb{R}$. Thus by Lemma 3.1, we obtain

$$
\begin{aligned}
I(t(v, 0)) & =\frac{t^{2}}{2} \int_{\mathbb{R}^{N}}|\nabla v|^{2}+a(x) v^{2} d x-\int_{\mathbb{R}^{N}} \int_{0}^{t v} f(x, \tau, 0) d \tau \\
& \leq \frac{t^{2}}{2} \int_{\mathbb{R}^{N}}|\nabla v|^{2}+a(x) v^{2} d x-\int_{\mathbb{R}^{N}} \int_{0}^{t v}\left(C_{1} \tau^{\mu-1}-C_{2}\right) d \tau \\
& =\frac{t^{2}}{2} \int_{\mathbb{R}^{N}}|\nabla v|^{2}+a(x) v^{2} d x+C_{2} t \int_{\mathbb{R}^{N}} v d x-C_{1}^{\prime} t^{\mu} \int_{\mathbb{R}^{N}} v^{\mu} d x<0
\end{aligned}
$$

for $t>0$ large enough.

Verification of (3.3). We observe that (1.14), (1.15), and (1.16) imply that for any $\varepsilon>0$, there exists a constant $A_{\varepsilon}>0$ such that

$$
\begin{aligned}
& |f(x, s)| \leq \varepsilon|s|+A_{\varepsilon}|s|^{p} \\
& |g(x, s)| \leq \varepsilon|s|+A_{\varepsilon}|s|^{p} \quad \text { for a.e. }(x, s) \in \mathbb{R}^{N} \times \mathbb{R}^{2} .
\end{aligned}
$$

By (3.7) and Sobolev's embedding theorem, we have for any $U \in E$,

$$
\begin{aligned}
|\Psi(U)|= & \left|\Psi\left(u_{1}, u_{2}\right)\right| \\
\leq & \int_{\mathbb{R}^{N}} \int_{0}^{\left|u_{1}\right|}\left|f\left(x, \tau, u_{2}\right)\right| d \tau+\int_{\mathbb{R}^{N}} \int_{0}^{u_{2}}|g(x, 0, \tau)| d \tau \\
\leq & \int_{\mathbb{R}^{N}}\left(\frac{\varepsilon}{2}\left|\left(u_{1}, u_{2}\right)\right|^{2}+\frac{A_{\varepsilon}}{p+1}\left|u_{1}, u_{2}\right|^{p+1}\right) d x \\
& +\int_{\mathbb{R}^{N}}\left(\frac{\varepsilon}{2}\left|u_{2}\right|^{2}+\frac{A_{\varepsilon}}{p+1}\left|u_{2}\right|^{p+1}\right) d x \\
\leq & \varepsilon\|U\|_{L^{2}}^{2}+\frac{2 A_{\varepsilon}}{p+1}\|U\|_{L^{p+1}}^{p+1} \\
\leq & \varepsilon C_{3}\|U\|_{E}^{2}+C_{4}\|U\|_{E}^{p+1}
\end{aligned}
$$

where $\varepsilon$ is arbitrary and $C_{4}=C_{4}(\varepsilon)$. Thus

$$
I(U)=\frac{1}{2}\|U\|_{E}^{2}-\Psi(U) \geq \frac{1}{2}\|U\|_{E}^{2}-\varepsilon C_{3}\|U\|_{E}^{2}-C_{4}\|U\|_{E}^{p+1} \geq \beta>0,
$$

for $\|U\|_{E}=\rho$, with $\rho, \varepsilon$, and $\beta$ sufficiently small positive constants.

Denote

$$
\begin{gathered}
\mathscr{P}=\{\gamma \in C([0,1], E) ; \gamma(0)=0, \gamma(1) \neq 0, I(\gamma(1)) \leq 0\}, \\
c=\inf _{\gamma \in \mathscr{P}} \max _{t \in[0,1]} I(\gamma(t)) .
\end{gathered}
$$

Set

$$
\lambda_{I}(U)=\min _{\xi \in \partial I(U)}\|\xi\|_{E^{*}}
$$


Thus, by the nonsmooth version of the mountain pass lemma [2], there exists a sequence $\left\{U_{M}\right\} \subset E$ such that

$$
I\left(U_{m}\right) \longrightarrow c, \quad \lambda_{I}\left(U_{m}\right) \longrightarrow 0 .
$$

So, there exists a sequence $\left\{W_{m}\right\} \subset \partial \Psi\left(U_{m}\right), W_{m}=\left(w_{m}^{1}, w_{m}^{2}\right)$, such that

$$
\left(-\Delta u_{m}^{1}+a(x) u_{m}^{1}-w_{m}^{1},-\Delta u_{m}^{2}+a(x) u_{m}^{2}-w_{m}^{2}\right) \longrightarrow 0 \quad \text { in } E^{*}
$$

Note that by (1.16),

$$
\begin{aligned}
& \Psi(U) \leq \frac{1}{\mu}\left(\int_{u_{1} \geq 0} u_{1}(x) \underline{f}(x, U) d x+\int_{u_{1} \leq 0} u_{1}(x) \bar{f}(x, U) d x\right. \\
& \left.\quad+\int_{u_{2} \geq 0} u_{1}(x) \underline{g}(x, U) d x+\int_{u_{2} \leq 0} u_{2}(x) \bar{g}(x, U) d x\right) .
\end{aligned}
$$

Therefore, by (2.31),

$$
\Psi(U) \leq \frac{1}{\mu} \int_{\mathbb{R}^{N}} U(x) W(x) d x=\frac{1}{\mu} \int_{\mathbb{R}^{N}}\left(u_{1} w_{1}+u_{2} w_{2}\right) d x
$$

for every $U \in E$ and $W \in \partial \Psi(U)$. Hence, if $\langle\cdot, \cdot\rangle$ denotes the duality pairing between $E^{*}$ and $E$, we have

$$
\begin{aligned}
I\left(U_{m}\right)= & \frac{\mu-2}{2 \mu} \int_{\mathbb{R}^{N}}\left(\left|\nabla u_{m}^{1}\right|^{2}+\left|\nabla u_{m}\right|^{2}+a(x)\left|u_{m}\right|^{1}+b(x)\left|u_{m}\right|^{2}\right) d x \\
& +\frac{1}{\mu}\left\langle\left(-\Delta u_{m}^{1}+a(x) u_{m}^{1}-w_{m}^{1},-\Delta u_{m}^{2}+b(x) u_{m}^{2}-w_{m}^{2}\right), U_{m}\right\rangle \\
& +\frac{1}{\mu}\left\langle W_{m}, U_{m}\right\rangle-\Psi\left(U_{m}\right) \\
\geq & \frac{\mu-2}{2 \mu} \int_{\mathbb{R}^{N}}\left(\left|\nabla u_{m}^{1}\right|^{2}+\left|\nabla u_{m}^{2}\right|^{2}+a(x)\left|u_{m}^{1}\right|^{2}+b(x)\left|u_{m}^{2}\right|^{2}\right) d x \\
& +\frac{1}{\mu}\left\langle\left(-\Delta u_{m}^{1}+a(x) u_{m}^{1}-w_{m}^{1},-\Delta u_{m}^{2}+b(x) u_{m}^{2}-w_{m}^{2}\right), U_{m}\right\rangle \\
\geq & \frac{\mu-2}{2 \mu}\left\|U_{m}\right\|_{E}^{2}-o(1)|| U_{m} \|_{E} .
\end{aligned}
$$

This relation in conjunction with (3.12) implies that the Palais-Smale sequence $\left\{U_{m}\right\}$ is bounded in $E$. Thus, it converges weakly (up to a subsequence) in $E$ and strongly in $L_{\text {loc }}^{2}\left(\mathbb{R}^{N}\right)$ to some $U$. Taking into account that $W_{m} \in \partial \Psi\left(U_{m}\right)$ and $U_{m} \rightarrow U$ in $E$, we deduce from (3.13) that there exists $W \in E^{*}$ such that $W_{m} \rightarrow W$ in $E^{*}$ (up to a subsequence). Since the mapping $U \mapsto F(x, U)$ is compact from $E$ to $L^{1}$, it follows that 
$W \in \partial \Psi(U)$. Therefore,

$$
\begin{gathered}
W(x) \in[\underline{f}(x, U(x)), \bar{f}(x, U(x))] \times[\underline{g}(x, U(x)), \bar{g}(x, U(x))] \quad \text { a.e. } x \text { in } \mathbb{R}^{N}, \\
\begin{array}{c}
\left(-\Delta u_{m}^{1}+a(x) u_{m}^{1}-w_{m}^{1},-\Delta u_{m}^{2}+b(x) u_{m}^{2}-w_{m}^{2}\right) \\
=0 \Longleftrightarrow \int_{\mathbb{R}^{N}}\left(\nabla u_{1} \nabla v_{1}+\nabla u_{2} \nabla v_{2}+a(x) u_{1} v_{1}+b(x) u_{2} v_{2}\right) d x \\
=\int_{\mathbb{R}^{N}}\left(w_{1} v_{1}+w_{2} v_{2}\right) d x \quad \forall\left(v_{1}, v_{2}\right) \in E .
\end{array}
\end{gathered}
$$

These last two relations show that $U$ is a solution of the problem (1.9).

It remains to prove that $U \not \equiv 0$. If $\left\{W_{m}\right\}$ is as in (3.13), then by (1.16), (2.31), (3.12), and for large $m$,

$$
\begin{aligned}
\frac{c}{2} \leq & I\left(U_{m}\right)-\frac{1}{2}\left\langle\left(-\Delta u_{m}^{1}+a(x) u_{m}^{1}-w_{m}^{1},-\Delta u_{m}^{2}+b(x) u_{m}^{2}-w_{m}^{2}\right), U_{m}\right\rangle \\
= & \frac{1}{2}\left\langle W_{m}, U_{m}\right\rangle-\int_{\mathbb{R}^{N}} F\left(x, U_{m}\right) d x \\
\leq & \frac{1}{2}\left(\int_{u_{1} \geq 0} u_{1}(x) \underline{f}(x, U) d x+\int_{u_{1} \leq 0} u_{1}(x) \bar{f}(x, U) d x\right. \\
& \left.+\int_{u_{2} \geq 0} u_{1}(x) \underline{g}(x, U) d x+\int_{u_{2} \leq 0} u_{2}(x) \bar{g}(x, U) d x\right) .
\end{aligned}
$$

Now, taking into account the definition of $\bar{f}, f, \bar{g}, g$, we deduce that $\bar{f}, f, \bar{g}, g$ verify (3.2) too. So by (3.18), we obtain

$$
\frac{c}{2} \leq \int_{\mathbb{R}^{N}}\left(\varepsilon\left|U_{m}\right|^{2}+A_{\varepsilon}\left|u_{m}\right|^{p+1}\right)=\varepsilon\left\|U_{m}\right\|_{L^{2}}^{2}+A_{\varepsilon}\left\|U_{m}\right\|_{L^{p+1}}^{p+1}
$$

So, $\left\{U_{m}\right\}$ does not converge strongly to 0 in $L^{p+1}\left(\mathbb{R}^{N} ; \mathbb{R}^{2}\right)$. From now on, with the same arguments as in the proof of $[6$, Theorem 1$]$, we deduce that $U \neq \equiv 0$, which concludes our proof.

\section{References}

[1] S. K. Berberian, Measure and Integration, The Macmillan, New York, 1967.

[2] K. C. Chang, Variational methods for nondifferentiable functionals and their applications to partial differential equations, Journal of Mathematical Analysis and Applications 80 (1981), no. 1, 102129.

[3] F. H. Clarke, Generalized gradients and applications, Transactions of the American Mathematical Society 205 (1975), 247-262.

[4] __ Generalized gradients of Lipschitz functionals, Advances in Mathematics 40 (1981), no. 1, $52-67$.

[5] B. D. Esry, C. H. Greene, J. P. Burke Jr., and J. L. Bohn, Hartree-Fock theory for double condensates, Physical Review Letters 78 (1997), no. 19, 3594-3597.

[6] F. Gazzola and V. Rădulescu, A nonsmooth critical point theory approach to some nonlinear elliptic equations in $\mathbb{R}^{n}$, Differential Integral Equations 13 (2000), no. 1-3, 47-60. 
[7] H. Grosse and A. Martin, Particle Physics and the Schrödinger Equation, Cambridge Monographs on Particle Physics, Nuclear Physics and Cosmology, vol. 6, Cambridge University Press, Cambridge, 1997.

[8] A. Hasegawa and Y. Kodama, Solitons in Optical Communications, Academic Press, California, 1995.

[9] M. N. Islam, Ultrafast Fiber Switching Devices and Systems, Cambridge University Press, New York, 1992.

[10] T. Kato, Remarks on holomorphic families of Schrödinger and Dirac operators, Differential Equations (Birmingham, Ala., 1983) (I. Knowles and R. Lewis, eds.), North-Holland Math. Stud., vol. 92, North-Holland, Amsterdam, 1984, pp. 341-352.

[11] P. Mironescu and V. Rădulescu, A multiplicity theorem for locally Lipschitz periodic functionals, Journal of Mathematical Analysis and Applications 195 (1995), no. 3, 621-637.

[12] Y.-G. Oh, Existence of semiclassical bound states of nonlinear Schrödinger equations with potentials of the class $\left(V_{a}\right)$, Communications in Partial Differential Equations 13 (1988), no. 12, 14991519.

[13] P. H. Rabinowitz, On a class of nonlinear Schrödinger equations, Zeitschrift für Angewandte Mathematik und Physik 43 (1992), no. 2, 270-291.

[14] C. Sulem and P.-L. Sulem, The Nonlinear Schrödinger Equation. Self-Focusing and Wave Collapse, Applied Mathematical Sciences, vol. 139, Springer, New York, 1999.

Teodora-Liliana Dinu: Department of Mathematics, "Fraţii Buzeşti” College, Boulevard Ştirbei-Vodă no. 5, Craiova 200352, Romania

E-mail address: tldinu@gmail.com 


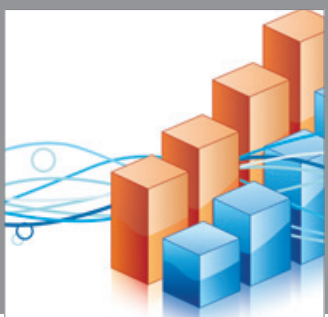

Advances in

Operations Research

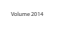

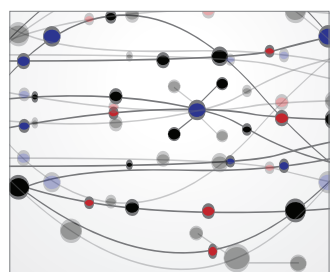

\section{The Scientific} World Journal
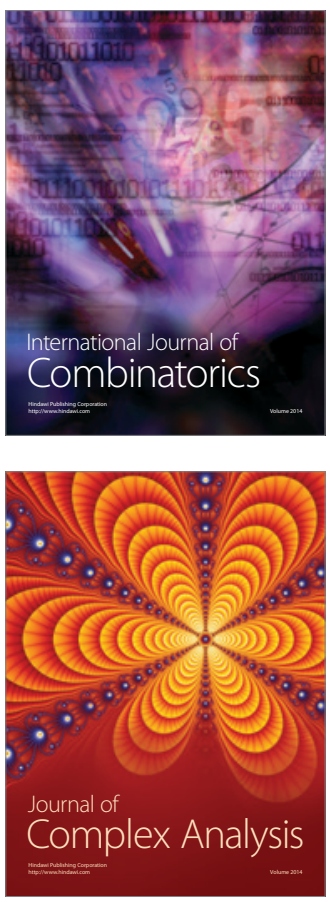

International Journal of

Mathematics and

Mathematical

Sciences
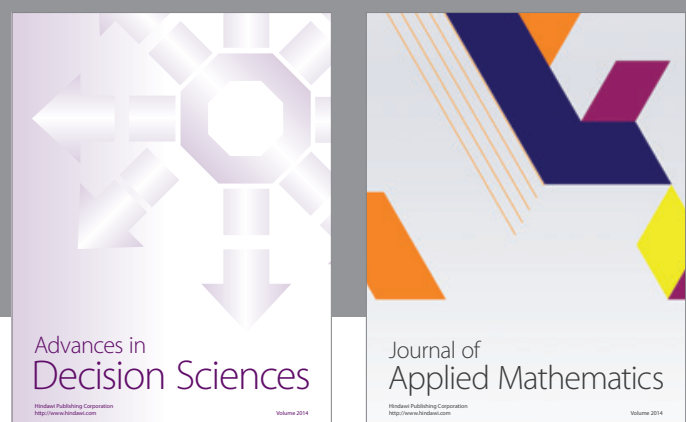

Journal of

Applied Mathematics
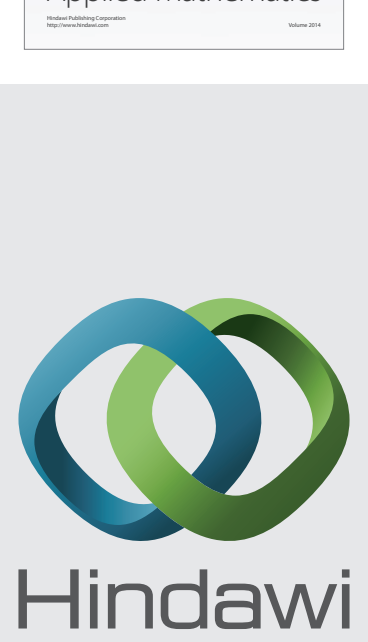

Submit your manuscripts at http://www.hindawi.com
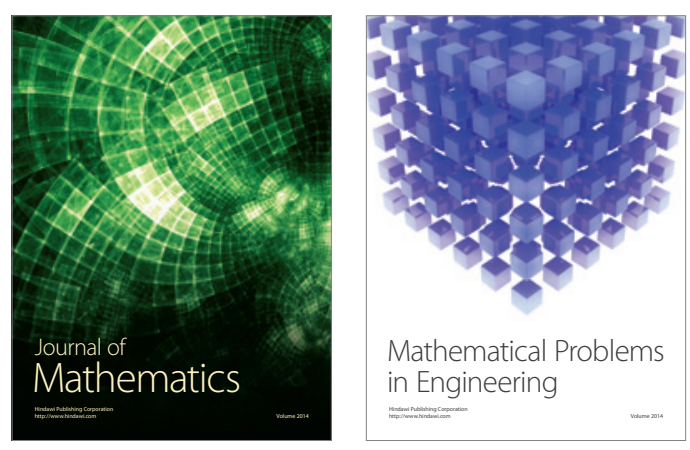

Mathematical Problems in Engineering
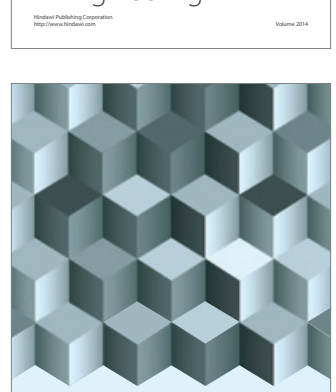

Journal of

Function Spaces
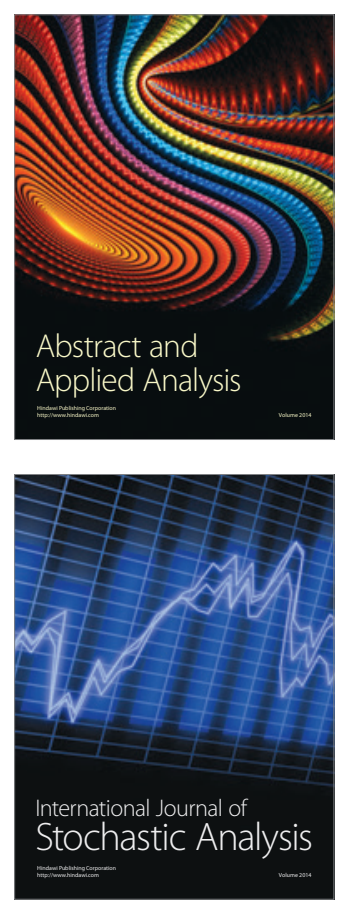

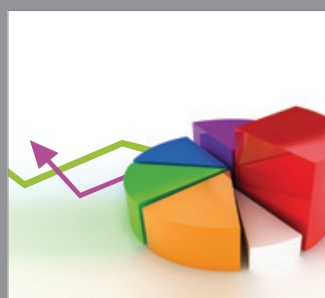

ournal of

Probability and Statistics

Promensencen
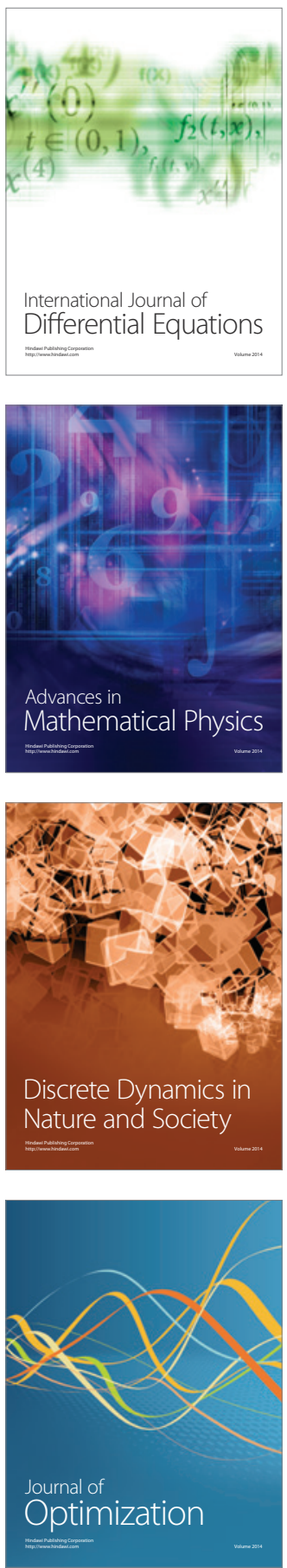